Archived version from NCDOCKS Institutional Repository http://libres.uncg.edu/ir/asu/

\title{
Appalachl̈ăn
}

B O O N E, N O R T H C A R O L I N A

\section{Hoping for more: \\ The influence of outcome desirability on information seeking and predictions about relative quantities.}

\author{
Authors \\ Scherer, A. M., Windschitl, P. D., O'Rourke, J., \& Smith, A. R.
}

\begin{abstract}
$\underline{\text { Abstract }}$
People must often engage in sequential sampling in order to make predictions about the relative quantities of two options. We investigated how directional motives influence sampling selections and resulting predictions in such cases. We used a paradigm in which participants had limited time to sample items and make predictions about which side of the screen contained more of a critical item. Sampling selections were biased by monetary desirability manipulations, and participants exhibited a desirability bias for both dichotmous and continuous predictions.
\end{abstract}

Scherer, A. M., Windschitl, P. D., O'Rourke, J., \& Smith, A. R. (2012) " Hoping for more: The influence of outcome desirability on information seeking and predictions about relative quantities" Cognition 125,pp. 113-117 (ISSN: 0010-0277) Version of Record available @ (http://dx.doi.org/10.1016/j.cognition.2012.06.013) 


\title{
Hoping for more: The influence of outcome desirability on information seeking and predictions about relative quantities
}

\author{
Aaron M. Scherer ${ }^{a, \Uparrow}$, Paul D. Windschitl ${ }^{\mathrm{a}}$, Jillian O’Rourke ${ }^{\mathrm{a}}$, Andrew R. Smith ${ }^{\mathrm{b}}$ \\ ${ }^{a}$ Department of Psychology, University of Iowa, Iowa City, IA 52241, United States \\ ${ }^{\mathrm{b}}$ Department of Psychology, Appalachian State University, Boone, NC 28608, United States
}

Keywords:

Selective exposure

Information seeking

Desirability bias

Wishful thinking

Overconfidence

\author{
$\mathrm{abstract}$
}

People must often engage in sequential sampling in order to make predictions about the relative quantities of two options. We investigated how directional motives influence sampling selections and resulting predictions in such cases. We used a paradigm in which participants had limited time to sample items and make predictions about which side of the screen contained more of a critical item. Sampling selections were biased by monetary desirability manipulations, and participants exhibited a desirability bias for both dichotmous and continuous predictions.

\section{Introduction}

People often make predictions about relative quantities. Information seeking can be a key step in the process. For example, to predict whether there is a higher proportion of gothic-style buildings at Princeton or Washington University, a person might sample internet pictures of buildings from both universities. There are a variety of biases-both cognitive and motivated - that can influence how people sample information for such predictions (for reviews, see Crocker, 1981; Fiedler, 2000; Klayman \& Ha, 1987; Trope \& Liberman, 1993). The present work concerns the potential role of a particular motivated bias tied to outcome desirability. In the context of our example, outcome desirability might refer to a desire that the proportion of gothic buildings is actually higher at Princeton.

Research in social psychology suggests that when information is threatening or reflects negatively on the self, people often avoid that information and/or seek more positive information (Festinger, 1957; Frey, 1986; Hart et al., 2009; Kunda, 1990; Pyszczynski \& Greenberg, 1987). However, in inference tasks for which available information is not directly self-relevant, it is unclear whether people would sample information in a manner biased by outcome desirability, and whether such a bias would then influence optimism (see Krizan \& Windschitl, 2007). In the present studies, we used a paradigm in which people made predictions about relative quantities after sampling information. The outcomes and to-be-sampled information were entirely novel and not substantially self-relevant to participants. However, we used monetary manipulations to make the outcomesabout which participants were making predictions-either desirable or undesirable.

The specific task faced by our participants involved virtual tiles (see Fig. 1). When sampling, participants saw 30 tiles on the left of their screen and 30 on the right. The top sides of all the tiles were identical, but for $12 \mathrm{~s}$ participants could turn over individual tiles to see whether their undersides displayed a particular target image. Then participants predicted whether, across all 60 tiles, there were 




Fig. 1. Black and white screenshot of the information sampling stage of the study.

more of these targets on the left or right. Critically, before the sampling started, we manipulated whether a participant would hope that more targets were on the left (or right). On some rounds, participants knew they would gain money if there were more targets on the left than right (otherwise they would gain nothing). We also had loss rounds where, for example, participants knew they would lose money if there were more targets on the left than right (otherwise they would lose nothing). Another important feature was that all participants had a strong monetary incentive-separate from the desirability manipulationsto form accurate predictions.

There were several plausible hypotheses on how our normatively irrelevant manipulations of outcome desirability would influence information sampling and predictions. Our leading hypothesis was that participants would exhibit a desirability bias (or wishful thinking) both in their sampling patterns and predictions (e.g., Krizan \& Windschitl, 2007; Lench, 2009). That is, they would sample in places where they hoped large numbers of targets to be-rather than sample evenly between the left and right locations. This hope should not be a reason to search more on one side than another, given what participants knew about how the distribution of tiles was determined (see details in Section 2.1). By sampling more on the desired side, they would tend to find more targets on that side even though the proportion of targets-to-not-targets found would be the same (on average) for the two sides. Having seen more targets on the desired side, they would then tend to be overoptimistic. In other words, they would tend to predict that, among all 60 tiles, there were more targets on the desired side of the screen.

Regarding alternative hypotheses, one might expect that participants would be realistic and unbiasedespecially because we used a strong accuracy incentive and be- cause participants assumed that accuracy feedback was impending rather than far into the future (see Carroll, Swe- eny, \& Shepperd, 2006 for a review). Another alternative hypothesis anticipates a more pessimistic pattern. It is inspired by work on the negativity bias (for reviews see Baumeister, Bratslavsky, Finkenauer, \& Vohs, 2001; Rozin \& Royzman, 2001) and suggests that people might sample more on the undesired side, perhaps as a way of bracing or ensuring that one isn't surprised by bad news (e.g., Sweeny \& Shepperd, 2010). Critically, we included loss rounds so that we could distinguish our desirability hypothesis from yet another alternative hypothesis inspired by Vosgerau's (2010) recent work on the stakes-likelihood hypothesis. Namely, perhaps participants would sample more from a high-stakes side rather than a side worth $\$ 0$ - regardless of whether the stakes are positive (e.g., potential to gain $\$ 5$ ) or negative (e.g., potential to lose $\$ 5$ ). Our desir- ability hypothesis and this stakes hypothesis predict the same results for gain rounds, but differing results for loss rounds-with the stakes hypothesis predicting that people would sample more from the undesirable side (e.g., lose

$\$ 5)$ than the neutral side $(\$ 0)$.

\section{Experiment 1}

\subsection{Method}

Initial instructions introduced the undergraduate participants $(N=34)$ to the tile prediction task, the sampling process, and the potential monetary outcomes that would be used during the rounds. An accuracy incentive was also introduced. Namely, participants were told that, at the end of the experiment, they would be asked to predict how much money they had earned or lost overall. ${ }^{1}$ If they were accurate, they would receive a $\$ 5$ bonus.

\footnotetext{
${ }^{1}$ Awarded earnings/losses for each round were based on whether a participants' predicted side was also the side for which the \% of targets among viewed tiles was higher.
} 
There were four rounds. Prior to each round, one of five images (e.g., an orange) was arbitrarily selected by the participant as the target image for the upcoming round. Then the participant saw 60 tiles with identical topsides. From the initial instructions, participants were told that a random but unknown number of tiles (between 0 and 60) would contain the target image, while the rest of the tiles would contain other images. The participant clicked a button to initiate random shuffling of tiles to the left and right, so that each side contained 30 tiles (see Fig. 1). Then participants were informed that they would win (lose) $\$ 5$ if there were more targets on one side of the screen, and that they would win (lose) $\$ 0$ if there were more targets on the other side of the screen. The order of the gain and loss rounds and the side of the screen that was made desirable were counterbalanced. Participants were instructed that if their final earnings were below $\$ 0$, they would not have to pay the researchers; they would simply leave the study with $\$ 0$.

After the tiles had been shuffled, participants had $12 \mathrm{~s}$ to sample-i.e., view images. Participants viewed the image on the underside of a tile by clicking on the tile. Once a new tile was clicked, the previously selected tile was turned back over, allowing for sequential viewing only. After $12 \mathrm{~s}$, participants were asked to predict which side of the screen actually contained more tiles with target images.

Feedback was not provided during the rounds. After all rounds, participants were asked to estimate their overall earnings. Participants were then paid-with a minimum payout of $\$ 3$.

It is important to note that although participants were informed that between 0 to 60 of the tiles would contain the target image, the true number of targets per side was kept constant. Namely, each side of the screen contained 18 targets (out of 30 tiles). We chose our instructions carefully, so that it would be clear to participants that they would be unable to infer the number of targets on the opposite side of the screen based on their observations from one side of the screen or from their experiences in previous rounds. Therefore, the optimal strategy for predicting which side of the screen contained more targets would involve sampling equally from the two sides of the screen.

\subsection{Results and discussion}

The counterbalance factors had no reliable effects, so the results we report are collapsed across those factors. Participants clicked an average of 24.4 tiles $(S D=4.2)$ per round. Biases in information selection (i.e., sampling) were calculated by dividing the number of clicks on the desirable side of the screen (gain $\$ 5$ side for the gain rounds; lose $\$ 0$ side for the loss rounds) by the total number of clicks. Values greater than 50\% indicate a bias towards the desirable side. Rather than revealing unbiased selections or a pattern consistent with pessimism/vigilance/ bracing, the results were consistent with the desirability hypothesis. Namely, participants demonstrated a significant selection bias, with $53.9 \%(S D=7.7 \%)$ of their clicks being on the desirable side of the screen, $t(33)=2.97, p<.01$. The magnitude of the bias was not significantly different between the gain $(M=54.4, S D=10.9)$ and loss rounds $(M=53.4, S D=10.6), t(33)=0.38, p=.71$. That is, the tendency to pick the desirable side was about as strong when the side was attractive because it involved a gain of $\$ 5$ as when it was desirable because it was the side that avoided losing $\$ 5$. This latter result is consistent with the desirability hypothesis rather than the stakes hypothesis, which would predict that on loss rounds, people would tend to oversample from the lose $\$ 5$ side (i.e., the undesirable side).

Participants' predictions (regarding which side had more targets) revealed a related pattern. Participants predicted the desirable side $65.4 \%$ of the time $(S D=$ $20.4 \%$ ), which is significantly greater than $50 \%$ and therefore reflects a desirability bias, $t(33)=4.41, p<.001$. The tendency to predict the desirable side did not significantly differ between the gain $(M=70.6, S D=30.4)$ and loss rounds $(M=60.3, S D=36.5), t(33)=1.13, p=.27$. This pat-

tern of results for predictions, like the pattern for information sampling, supports the desirability hypothesis and not the stakes hypothesis (nor a pessimism hypothesis).

Additionally, selection biases and predictions were correlated for the gain $(r=.34, p<.05)$ and loss rounds $(r=.38$, $p<.05)$. In other words, greater desirability biases in sampling were associated with more optimistic predictions. Note it is not inevitable that sampling biases favoring the desirable side would lead to optimistic predictions. Participants could sample more from one side, but then mentally compute a targets-to-total-clicked-tiles ratio for each side. By comparing those ratios, participants' resulting predictions would be unbiased (but could be quite noisy). The fact that participants were overly optimistic in their pre- dictions and that this tendency was related to the selection biases suggests that participants were at least partially influenced by the absolute frequency of the targets seen on one side versus another (see Estes, 1976).

Finally, participants were also overoptimistic in their predictions about their final earnings. While their prediction should have averaged around $\$ 0$, the average predicted earnings was $\$ 5.74 \quad(S D=\$ 5.10)$, $t(33)=6.56, p<.001$.

\section{Follow-up to Experiment 1}

In response to a reviewer's concern about whether participants would believe that losses are plausible (given that they started with $\$ 0$ ), we conducted a new version of Experiment 1 but endowed participants $(N=47)$ with $\$ 6$ at the beginning of the study and included two rather than four rounds. We also tested a simplified accuracy incentive in which participants were informed that they would receive $\$ 1$ for each correct prediction in each round.

The results supported the same conclusions as discussed for Experiment 1. Participants again sampled more from the desirable side of the screen $(M=55.0, S D=12.2)$, $t(46)=2.78, p=.008$. This selection bias did not significantly differ between the gain $(M=56.8, S D=$ 17.4) and loss rounds $(M=53.1, S D=16.7), t(46)=1.06$, $p=.30$. Participants also again exhibited a significant desirability bias in their predictions $(M=64.9, \quad S D=31.1)$, $t(46)=3.30$, 
$p=.002$. Unlike Experiment 1, the predictions for the gain $(M=76.6, S D=42.8)$ and loss $(M=53.2, S D=50.4)$ rounds were significantly different, $t(46)=2.30, p=.03$, but both means were again in a direction consistent with the desirability hypothesis. The selection biases and predictions were marginally correlated for the gain round $(r=.25, p<.09)$ and significantly correlated for the loss round $(r=.49, p=.001)$. Finally, when giving total estimates regarding how much they would gain/lose from the out- comes of the two rounds, which on average should be $\$ 0$, participants' average estimates were overoptimistic $(\$ 3.85 ; S D=\$ 3.56), t(46)=7.42, p<$ .001 .

\section{Experiment 2}

Experiment 1 and its follow-up revealed that, when participants had to make a dichotomous prediction (left or right), both sampling and predictions were influenced by desirability. Previous research, which did not involve sampling processes, has revealed that although desirability biases can be detected when people are asked to make dichotomous predictions, the biases often vanish for likelihood judgments or more continuous predictions (see Windschitl, Smith, Rose, \& Krizan, 2010; also see Dai, Wertenbroch, \& Brendl, 2008 for a reversal). Because information sampling is possible and can be biased in the current paradigm (i.e., participants are not merely given a fixed set of information), we expected to find desirability biases even when continuous scales are used to solicit predictions. This was tested in Experiment 2. Finding a desirability bias on continuous scales of likelihood or of prediction confidence is something that has been considered "elu- sive" in the wishful thinking literature (Bar-Hillel \& Bude- scu, 1995; see also Krizan \& Windschitl, 2007). We also made minor changes to further test the generalizability of the Experiment 1 results. Namely, we varied the number of targets across rounds and added variety to the dollar amounts used.

\subsection{Method}

The method was similar to that for Experiment 1, except as follows. Besides counterbalancing, the design was a 2 (gain or loss rounds) $\times 3(6,18$, or 24 targets per side) $\times 2$ (dichotomous or continuous prediction) mixed- factor design. Each participant $(N=87)$ completed six rounds. Instead of always presenting $\$ 5$ as the amount to be gained or lost during the gain and loss rounds, values of $\$ 3, \$ 4$, or $\$ 5$ were used. As expected, this small variety in dollar amounts did not impact results and will not be discussed further. The gain-loss factor was crossed by whether there were 6,18 , or 24 targets per side, out of 30 tiles per side. (As in Experiment 1, participants were told that the total numbers of targets was randomly deter- mined between 0 and 60.) Some participants were asked to make a dichotomous prediction in each round, as in Experiment 1. Others were asked to indicate their prediction/ confidence on a continuous, visual-analog scale with the anchors of Definitely more of the target image on the left and Definitely more of the target image on the right.

\subsection{Results and discussion}

The overall results were similar to those from Experiment 1. On average, participants clicked 25.7 tiles $(S D=4.5)$. Participants again exhibited a significant selection bias favoring the desirable side $(M=$ $53.3 \%, S D=10.5), t(86)=2.88, p<.01$. This bias did not significantly differ as a function of the counterbalancing factors $(p s>.09)$, nor as a function of the number-oftargets or prediction-type factors $(p s=.22)$. The bias also did not significantly differ between the gain $(M=54.0, S D$ $=12.4)$ and loss rounds $(M=52.5, S D=11.9), t(86)=1.21$, $p=.23$.

The predictions also revealed desirability biases. Participants making dichotomous predictions indicated the desirable side more than $50 \%$ of the time (specifically $56.5 \% ; S D=17.0), t(45)=2.60, p<.05$. For participants using a continuous scale, we coded responses such that 100 (0) would reflect maximal certainty that there were more targets on the desirable (undesirable) side of the screen. The average of these responses $(M=55.3 ; S D$ $=15.0$ ) was significantly greater than 50, which also reveals a desirability bias, $t(40)=2.27, p<.05$. These desirability biases in predictions were not meaningfully qualified by the other main factors in the design. ${ }^{2}$

Consistent with Experiment 1, the more biased participants were in their information search the more likely they were to predict that the desirable side had more targets. This was true for dichotomous (gain: $r=.50$, $p<.001$; loss: $r=.30, p<.05$ ) and continuous (gain: $r=$ $.35, p<.01$; loss: $r=.42, p<.01)$ predictions.

Finally, participants were again overoptimistic in their final earnings predictions. When estimating their final earnings, their estimate also included a filler round (not mentioned above) that had an average payout of $-\$ 2$ so their overall estimate should have averaged around $-\$ 2$. However, it was significantly higher than that $(M=\$ 4.25$, $S D=\$ 4.65), t(86)=12.53, p<.001$.

\section{General discussion}

There were four theoretically plausible data patterns for how outcome desirability would influence information sampling and predictions in our studies. One possibility was that participants would be unbiased and realistic given the possibility for an accuracy bonus and that accuracy feedback was impending rather than distant (see Carroll et al., 2006). A second possibility was that, because of a negativity bias or bracing for bad news, participants would be inclined to check on pessimistic news by examining the undesired side (e.g., Rozin \& Royzman, 2001; Sweeny \& Shepperd, 2010). A third possibility was that participants would tend to seek information about outcomes with high- er stakes, even when those stakes involve losses (Vosgerau, 2010). The observed results, however, supported the desirability hypothesis. Participants were modestly but reliably biased toward seeking information on the desirable side and were biased in an optimistic direction regarding their

\footnotetext{
${ }^{2}$ There was a three- and four-way interaction that could not be meaningfully interpreted. Otherwise, all ps $>.19$
} 
outcome predictions. The results are corroborated by recent (unpublished) findings from our lab showing the influence of outcome desirability on information seeking in a different type of paradigm (see Windschitl, Scherer, Smith, \& Rose, 2012). Importantly, we have demonstrated that the influences of motivated biases in information sampling are not restricted to cases in which the information substantially reflects on the self (such as attitude-challenging information or threatening health information). Furthermore, our desirability manipulations were aimed at entirely novel outcomes, precluding the role of confounds that can accompany some desirability manipulations, such as a priori expectations (for discussion see Krizan \& Winds- chitl, 2007).

The desirability biases observed here are clearly related to, but distinct from, positive test and focalism biases (Fox \& Levav, 2000; Klayman \& Ha, 1987; McKenzie, 1998; Windschitl, 2000). These latter biases are nonmotivated. They refer to how people seek and consider information about a focal hypothesis (or outcome)-regardless of the desirability of that hypothesis. In our work, we did not designate a focal outcome. It is possible, however, that the desirability of an outcome causes people to essentially adopt an outcome as focal, at which point positive test strategies and focalism have their influence. If so, our work can be thought of as showing that people adopt desirable outcomes, rather than undesirable ones or higher-stakes ones, as focal. Additional research should be aimed at the nexus of directional motivated biases and non-motivated processes to arrive at a more comprehensive view of how people sample information for making various predictions in everyday contexts.

\section{Acknowledgements}

This work was supported by Grant SES 07-20349 and 09-61252 to Paul D. Windschitl from the National Science Foundation.

\section{References}

Bar-Hillel, M., \& Budescu, D. (1995). The elusive wishful thinking effect. Thinking and Reasoning, 1, 71-103. http://dx.doi.org/10.1080/ 13546789508256906 .

Baumeister, R. F., Bratslavsky, E., Finkenauer, C., \& Vohs, K. D. (2001). Bad is stronger than good. Review of General Psychology, 5, 323-370. http://dx.doi.org/10.1037/1089-2680.5.4.323.

Carroll, P., Sweeny, K., \& Shepperd, J. A. (2006). Forsaking optimism. Review of General Psychology, 10, 56-73. http://dx.doi.org/10.1037/ 1089-2680.10.1.56

Crocker, J. (1981). Judgment of covariation by social perceivers. Psychological Bulletin, 90, 272-292. http://dx.doi.org/10.1037/00332909.90.2.272.

Dai, X., Wertenbroch, K., \& Brendl, C. M. (2008). The value heuristic in judgments of relative frequency. Psychological Science, 19, 18-19. http://dx.doi.org/10.1111/j.1467-9280.2008.02039.x.
Estes, W. K. (1976). The cognitive side of probability learning. Psychological Review, 83, 37-64. http://dx.doi.org/10.1037/0033295X.83.1.37.

Festinger, L. (1957). Theories of cognitive dissonance. Stanford, CA: Stanford University Press.

Fiedler, K. (2000). Beware of samples! A cognitive-ecological sampling approach to judgment biases. Psychological Review, 107, 659-676. http://dx.doi.org/10.1037/0033-295X.107.4.659.

Fox, C. R., \& Levav, J. (2000). Familiarity bias and belief reversal in relative likelihood judgment. Organizational Behavior and Human Decision Processes, 82, 268-292. http://dx.doi.org/10.1006/obhd.2000.2898.

Frey, D. (1986). Recent research on selective exposure to information. In L. Berkowitz (Ed.). Advances in experimental social psychology (Vol. 19, pp. 41-80). San Diego, CA, US: Academic Press.

Hart, W., Albarracín, D., Eagly, A. H., Brechan, I., Lindberg, M. J., \& Merrill, L. (2009). Feeling validated versus being correct: A meta-analysis of selective exposure to information. Psychological Bulletin, 135, 555588. http://dx.doi.org/10.1037/a0015701.

Klayman, J., \& Ha, Y.-won. (1987). Confirmation, disconfirmation, and information in hypothesis testing. Psychological Review, 94, 211-228. http://dx.doi.org/10.1037/0033-295X.94.2.211.

Krizan, Z., \& Windschitl, P. D. (2007). The influence of outcome desirability on optimism. Psychological Bulletin, 133, 95-121. http:// dx.doi.org/10.1037/0033-2909.133.1.95.

Kunda, Z. (1990). The case for motivated reasoning. Psychological Bulletin, 108, 480-498. http://dx.doi.org/10.1037/0033-2909.108.3.480.

Lench, H. C. (2009). Automatic optimism: The affective basis of judgments about the likelihood of future events. Journal of Experimental Psychology: General, 138, 187-200. http://dx.doi.org/10.1037/ a0015380.

McKenzie, C. R. M. (1998). Taking into account the strength of an alternative hypothesis. Journal of Experimental Psychology: Learning, Memory, and Cognition, 24, 771-792. http://dx.doi.org/10.1037/02787393.24.3.771.

Pyszczynski, T., \& Greenberg, J. (1987). Towards an integration of cognitive and motivational perspectives on social inference: A biased hypothesis-testing model. In L. Berkowitz (Ed.). Advances in Experimental Social Psychology (Vol. 20, pp. 297-340). San Diego, CA, US: Academic Press.

Rozin, P., \& Royzman, E. B. (2001). Negativity bias, negativity dominance, and contagion. Personality and Social Psychology Review, 5, 296-320. http://dx.doi.org/10.1207/S15327957PSPR0504_2.

Sweeny, K., \& Shepperd, J. A. (2010). The costs of optimism and the benefits of pessimism. Emotion, 10, 750-753. http://dx.doi.org/ 10.1037/a0019016.

Trope, Y., \& Liberman, A. (1993). The use of trait conceptions to identify other people's behavior and to draw inferences about their personalities. Personality and Social Psychology Bulletin, 19, 553-562. http://dx.doi.org/10.1177/0146167293195007.

Vosgerau, J. (2010). How prevalent is wishful thinking? Misattribution of arousal causes optimism and pessimism in subjective probabilities. Journal of Experimental Psychology: General, 139, 32-48. http:// dx.doi.org/10.1037/a0018144.

Windschitl, P. D. (2000). The binary additivity of subjective probability does not indicate the binary complementarity of perceived certainty. Organizational Behavior and Human Decision Processes, 81, 195-225. http://dx.doi.org/10.1006/obhd.1999.2876.

Windschitl, P. D., Scherer, A. M., Smith, A. R., Rose, J. P. (2012). Why so confident? The effects of outcome desirability on selective exposure and likelihood judgments. Unpublished manuscript. Department of Psychology, University of Iowa, Iowa City, IA, United States.

Windschitl, P. D., Smith, A. R., Rose, J. P., \& Krizan, Z. (2010). The desirability bias in predictions: Going optimistic without leaving realism. Organizational Behavior and Human Decision Processes, 111, 3347. doi: org/10.1016/j.obhdp.2009.08.003. 